Autoregulatory gene therapy for obesity

\title{
A fine balance: an autoregulatory gene therapy approach to treat obesity and achieve energy homeostasis
}

SL Samson, M Kohjima and L Chan

Gene Therapy (2009) 16, 1175-1177; doi:10.1038/gt.2009.88; published online 23 July 2009

Homeostatic systems exist in higher organisms to maintain weight stability through a balance between food intake and energy expenditure. However, our 'Western' lifestyle has prevailed over these mechanisms such that the prevalence of obesity has increased to pandemic levels and associated with this there has been a parallel escalation in the prevalence of type II diabetes. ${ }^{1}$ The acknowledged consequences of these diseases are increased morbidity and mortality, especially from cardiovascular disease, and an overwhelming financial burden on health care. Lifestyle changes (diet and exercise) or bariatric surgery can have some impact, but disappointingly few individuals are successful in achieving or maintaining weight loss. ${ }^{2}$ Pharmacologic therapies have been met with limited success or had additional obstacles, such as adverse effects, that make them less palatable. ${ }^{3}$ The recent report by Cao et al. ${ }^{4}$ presents an alternative proof-ofconcept approach to attain weight reduction and energy balance in mouse models of obesity. They have used gene therapy technology in the central nervous system (CNS) to express a potent neuropeptide, brain-derived neurotrophic factor (BDNF), to induce weight loss and improve glucose homeostasis in obese mice. However, they have coupled this with a novel autoregulatory component using micro-RNA (miRNA) to BDNF, which relies on physiologic signals to determine when weight control is achieved and to prevent further uncontrolled weight loss and cachexia.

The regulation of body weight and energy homeostasis is overseen by the CNS, specifically the hypothalamus and brain stem. ${ }^{5}$ The nutrient status of the body is communicated by a plethora of peptides, mostly released from fat (For example, leptin) or the gastrointestinal system (For example, ghrelin, insulin, PYY, oxyntomodulin). ${ }^{2}$ These peripheral signals are able to reach the hypothalamus through a permissive bloodbrain barrier or peptide-specific transport mechanisms. Their information is integrated in specific hypothalamic collections of neurons (nuclei), which send signals to the cerebral cortex and brainstem, promoting behaviors that affect food intake and energy expenditure. For example, a key peripheral peptide is leptin, which is released in proportion to fat mass and indicates that food intake should decrease to prevent further weight gain. This anorexigenic signal reaches the arcuate nucleus (ARC) to inhibit appetite-stimulating orexigenic neurons, which express NPY, agoutirelated protein (AgRP) and GABA. At the same time, there is activation of anorexigenic neurons, which express the pro-opiomelanocortin (POMC) peptide. POMC is processed to produce $\alpha$ - and $\beta$-melanocyte-stimulating hormones, which act on melanocortin receptors in the paraventricular nucleus and additional hypothalamic and brainstem regions to decrease appetite and increase energy expenditure. A proportion of the paraventricular nucleus neurons contain BDNF, which operates downstream of melanocyte-stimulating hormone, and is the neuropeptide of interest in the study by Cao et al. ${ }^{4}$

The centralized regulation of energy homeostasis in the CNS provides an attractive target for gene transfer therapy for obesity. Systemic gene therapy approaches may not be potent enough to have effects at the hypothalamic level. For example, despite the exciting potential of leptin at the time of its discovery, its promise as an anti-obesity treatment was short-lived because of its inability to affect CNS appetite centers due to 'leptin resistance,' which occurs with obesity. ${ }^{6}$ To circumvent this problem, one solution is to directly target the CNS using 'neurotherapy' where gene therapy approaches are attractive, avoiding the impracticality of multiple CNS injections or continuous infusions. Also, because so many of the known regulatory molecules are peptides, they can be expressed from their cDNA for continuous production. The replication-deficient adeno-associated virus (AAV) has been the vector of choice for animal studies. AAV serotypes 2, 3, and 5 have tropism for neuronal tissue and transduce brain cells when directly injected into the CNS. ${ }^{7}$ Stereotactic injection of AAV into the third cerebral ventricle, adjacent to the hypothalamus, or into the nuclei themselves have been used successfully to express leptin, POMC and leukemia inhibitory factor resulting in weight loss in rodent models of obesity. ${ }^{8,9}$ Alternatively, inhibition of the orexigenic peptide expression, such as $\mathrm{AgRP}^{10}$ has been achieved through RNA interference.

The early part of the study by Cao et al. ${ }^{4}$ mirrors the design of these previous CNS gene therapy reports, but with hypothalamic AAV-derived expression of BDNF. They arrived at this candidate neuropeptide rationally, based on their previous observations that housing mice in an enriched environmental and social setting not only was neuroprotective, but serendipitously resulted in decreased weight gain and improved glucose homeostasis over time. ${ }^{11}$ The improvements were also accompanied by an increase in BDNF expression in the hypothalamic ARC. Furthermore, others had shown that the loss of just a single BDNF allele led to hyperphagia and obesity. ${ }^{12,13}$ With this knowledge in hand, they used direct hypothalamic injection of AAV to express BDNF using the constitutively active cytomegalovirus enhancer-chicken $\beta$-actin promoter. ${ }^{4}$ Expression was achieved in AgRP/NPY neurons, and the result was significant loss of weight and fat mass in normal chow-fed mice, with a greater than $90 \%$ decrease in 


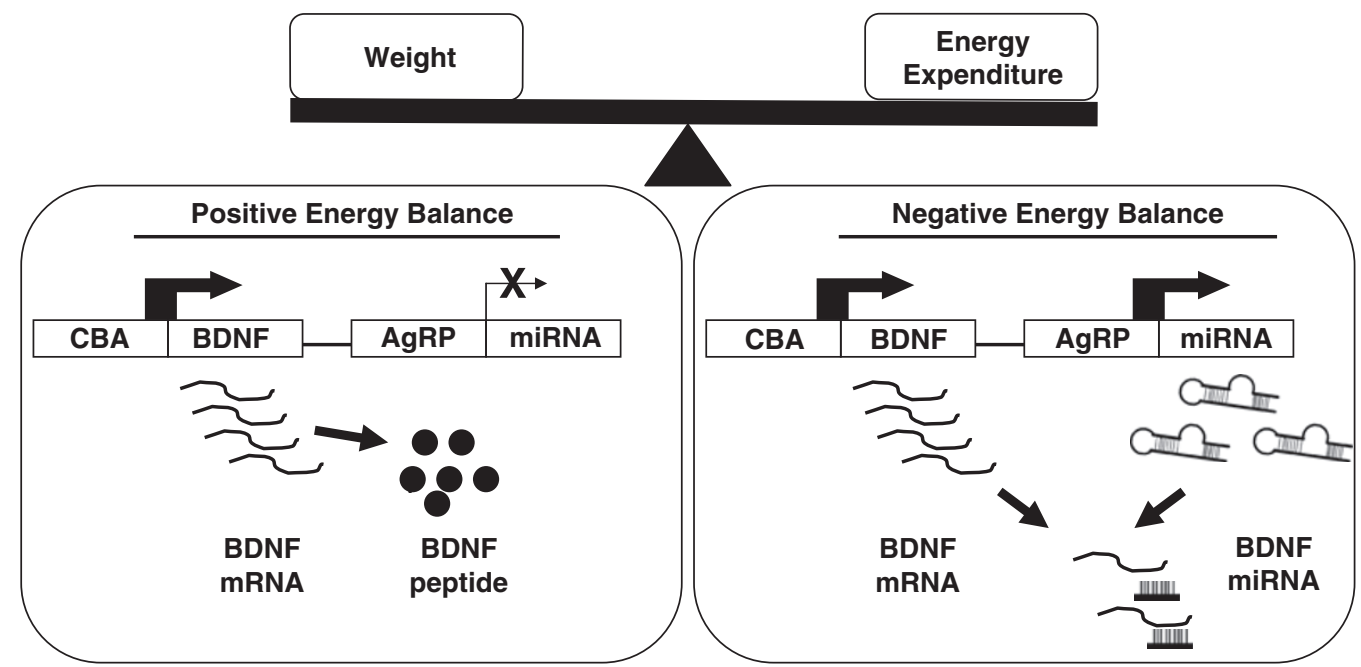

Figure 1 The design of the autoregulatory system by Cao et al. ${ }^{4}$ for treatment of obesity. The constitutive CMV-chicken $\beta$-actin promoter drives expression of BDNF to induce weight loss. As weight loss continues, the AgRP promoter is activated as a normal physiologic response, transcribing an miRNA to BDNF, which decreases BDNF peptide synthesis through translational repression and mRNA degradation.

perigonadal fat compared with controls by day 50 . However, there was no plateau in weight loss to the end of the study, indicating that the system could cross over to pathological weight loss. In fact, in the obese leptin-receptor-deficient $d b / d b$ mouse model, they mention the need to euthanize the animals due to wasting if BDNF expression continued unchecked. These experiments confirmed that BDNF has potential for gene therapy for obesity, but also that a major risk of this approach is that continued and unregulated expression of a potent weight lossinducing neuropeptide can induce unhealthy wasting or cachexia.

To address this, the researchers exploited a second important observation. Accompanying the BDNFinduced weight loss, there was a 15-fold increase in AgRP gene expression in the neurons, likely a physiologic but futile attempt to combat further decreases in weight. The single AAV-BDNF construct was modified by downstream addition of a cassette containing the sequence for an interfering miRNA under the regulation of the weight loss responsive AgRP promoter, reducing BDNF protein levels in the hypothalamus by $50 \%$. When the mice were in positive energy balance (gaining weight), BDNF was expressed unfettered from the constitutive chicken $\beta$-actin promoter, resulting in weight loss in $d b / d b$ mice. As further weight loss continued, compensatory activation of the AgRP promoter occurred to drive expression of the miRNA and downregulate BDNF peptide synthesis. With this modification, the system was now reliant on the neurons' own abilities to sense energy balance and respond. This resulted in more moderate weight loss, compared with a construct expressing scrambled miRNA, and there was a plateau in weight before pathologic effects could occur. Presumably, this approach led to oscillations in BDNF, with high levels when the animal is on a trajectory for weight gain and then low levels with the resultant weight loss (Figure 1).

A few regulatable gene therapy systems are available, including tetracycline-controlled systems ('Teton' and 'Tet-off'), rapamycin-FK506, and progesterone receptor/RU486. ${ }^{14}$ However, the authors rightly point out the limitations of these exogenously regulated systems for CNS gene therapy, as well as later clinical applications. The concept behind the autoregulatable system designed by Cao et al. ${ }^{4}$ could have wider applications to gene therapy beyond treatment for obesity, to be used wherever the level of a transgene product requires a natural 'brake', comprised of a suitable and physiologic counter-regulatory promoter to drive miRNA expression. Our knowledge of the repertoire of regulatory peptides for appetite and energy expenditure has increased exponentially over the last decade and there are numerous peptides known to decrease appetite and/or increase metabolic rate, and which could have potential for a similar autoregulatory approach in the hypothalamus. $^{2,5}$ Overall, for those of us in the field of obesity and molecular therapy, the investigators have left us satisfied, but with an appetite for more.

\section{Conflict of interest}

The authors declare no conflict of interest.

Drs L Chan, SL Samson and M Kohjima are at the Diabetes and Endocrinology Research Center, Baylor College of Medicine, One Baylor Plaza, Houston, TX 77030, USA.

E-mail:lchan@bcm.edu

1 Hossain P, Kawar B, Nahas ME. Obesity and diabetes in the developing world-a growing challenge. $N$ Engl J Med 2007; 356: 213-215.

2 Jayasena CN, Bloom SR. Role of gut hormones in obesity. Endocrinol Metab Clin North Am 2008; 37: 769-787.

3 Aronne LJ. Therapeutic options for modifying cardiometabolic risk factors. Am J Med 2007; 120: S26-S34.

4 Cao L, Lin EJD, Cahill MC, Wang CS, Liu XL, During MJ. Molecular therapy of obesity and diabetes by a physiological autoregulatory approach. Nature Med 2009; 15: 447-454.

5 Gao Q, Horvath TL. Neuronal control of energy homeostasis. FEBS Lett 2008; 582: 132-141.

6 Scarpace PJ, Zhang Y. Leptin resistance: a prediposing factor for diet-induced obesity. Am J Physiol Regul Integr Comp Physiol 2009; 296: R493-R500.

7 Shevtsova Z, Malik JMI, Michel U, Bahr M, Kugler S. Promoters and serotypes: 
targeting of adeno-associated virus vectors for gene transfer in the rat central nervous system in vitro and in vivo. Exp Physiol 2005; 90: 53-59.

8 Beretta E, Dhillon H, Kalra PS, Kalra SP. Central LIF gene therapy suppresses food intake, body weight, serum leptin and insulin for extended periods. Peptides 2002; 23: 975-984.

9 Zhang Y, Scarpace PJ. Circumventing central leptin resistance: lessons from central leptin and POMC gene delivery. Peptides 2006; 27: 350-364.

10 Makimura H, Mizumo TM, Mastaitis JW, Agami R, Mobbs CV. Reducing hypothalamic AGRP by RNA interfer- ence increases metabolic rate and decreases body weight without influencing food intake. BMC Neurosci 2002; 3: 18-23.

11 Young D, Lawlor PA, Leone P, Dragunow M, During MJ. Environmental enrichment inhibits spontaneous apoptosis, prevents seizures and is neuroprotective. Nature Med 1999; 5: 448-453.

12 Gray J, Yeo GSH, Cox JJ, Morton J, Adlam ALR, Keogh JM et al. Hyperphagia, severe obesity, impaired cognitive function, and hyperactivity associated with functional loss of one copy of the brain-derived neurotrophic factor
(BDNF) gene. Diabetes 2006; 55 3366-3371.

13 Lyons WE, Mamounas LA, Ricaurte GA Coppola V, Reid SW, Bora SH et al. Brain-derived neurotrophic factor-deficient mice develop aggressiveness and hyperphagia in conjunction with brain serotonergic abnormalities. Proc Natl Acad Sci U S A 1999; 96: 15239-15244.

14 Goverdhana S, Puntel M, Xiong W, Zirger JM, Barcia C, Curtin JF et al. Regulatable gene expression systems for gene therapy applications: progress and future challenges. Mol Ther 2005; 12: 189-211. 